\title{
A Robust and Low Frequency Stable Time Domain PMCHWT Equation
}

\author{
Y. Beghein* \\ K. Cools ${ }^{\dagger}$ \\ F.P. Andriulli ${ }^{\ddagger}$
}

\begin{abstract}
The time domain PMCHWT equation models transient scattering by piecewise homogeneous dielectrics. After discretization, it can be solved using the marching-on-in-time algorithm. Unfortunately, the PMCHWT equation suffers from DC instability: it supports constant in time regime solutions. Upon discretization, the corresponding poles of the system response function shift into the unstable region of the complex plane, rendering the MOT algorithm unstable. Furthermore, the discrete system becomes ill-conditioned when a large time step is used. This phenomenon is termed low frequency breakdown. In this contribution, the quasiHelmholtz components of the PMCHWT equation are separated using projector operators. Judicially integrating or differentiating these components of the basis and testing functions leads to an algorithm that (i) does not suffer from unstable modes even in the presence of moderate numerical errors, (ii) remains well-conditioned for large time steps, and (iii) can be applied effectively to both simply and multiply connected geometries.
\end{abstract}

\section{INTRODUCTION}

The time domain Poggio-Miller-Chan-HarringtonWu-Tsai (TD-PMCHWT) equation models transient scattering by piecewise homogeneous dielectrics. Like the time domain electric field integral equation (TD-EFIE), which is applicable to perfect conductors, it can be solved using the marching-on-in-time (MOT) algorithm. For both equations, this solution method suffers from low frequency breakdown and DC instability.

For slowly varying electromagnetic fields, the electro(quasi)static and magneto(quasi)static problems are decoupled. In both the TD-EFIE and the TD-PMCHWT equation, the two problems are lumped together, but with different frequency scaling. Upon discretization, this leads to an illconditioned MOT system when the time step is large. As a result, the system cannot be solved efficiently. This is low frequency breakdown.

DC instability originates from the existence of sourceless regime solutions to the TD-EFIE and the TD-PMCHWT equation, which inevitably show up

\footnotetext{
*Department of Information Technologoy (INTEC), Ghent University, Ghent, Belgium, e-mail: yves. beghein@ugent.be.

$\dagger$ Electrical Systems and Optics Division, University of Nottingham, Nottingham, U.K.

$¥$ Microwave Department of Telecom Bretagne, Institut Mines-Telecom, Brest, France.
}

in the numerical solution. In the case of the TDEFIE, these spurious currents are static (i.e., constant or at most linear in time). This is true even when the interaction integrals are computed with limited precision, because the spatial and temporal differentiations that lead to the cancellations of such solutions appear explicitly in the equation and can be performed up to machine precision. The TD-PMCHWT equation on the other hand is less robust: in the presence of even small quadrature errors, the - theoretically static - regime solutions become exponentially increasing. As a result, standard TD-PMCHWT MOT simulations can only be stable if all interaction integrals are computed with extremely high accuracy.

In [1], the quasi-Helmholtz projected TD-EFIE or qHP-TDEFIE has been introduced. The projector operators defined in [2] are applied to the TD-EFIE in order to separate its quasi-Helmholtz (i.e., loop and star) components without resorting to an explicit loop/star decomposition. These components are then differentiated or integrated with respect to time, and separately discretized in time using suitable temporal Galerkin methods. In this way, both DC instability and low frequency breakdown are solved for the TD-EFIE. This approach is applicable to both simply and multiply connected geometries, without having to explicitly deal with global topological loops.

The stabilization method of [1] cannot directly be applied to the TD-PMCHWT equation. The reason for this is that while the TD-EFIE operator cannot distinguish global from local loop currents, the TD-PMCHWT operator does discriminate between them. Therefore, an alternative stabilization method is developed in this contribution. The resulting equation, termed the qHP-PMCHWT equation, is immune to both DC instability and low frequency breakdown, and can directly be applied to both simply and multiply connected geometries. Most importantly, the proposed equation is robust: the resulting MOT scheme is stable even in the presence of moderate numerical errors.

This paper is organized as follows. In Section 2, the TD-PMCHWT is discretized in space. In Section 3 , the resulting semi-discrete equation is further manipulated in order to obtain a DC stable and low frequency stable equation, which is then 
discretized in time in Section 4. Finally, numerical results are presented in Section 5.

\section{SPATIAL DISCRETIZATION}

Consider a dielectric body $\Omega$ with boundary $\Gamma$ and exterior normal vector $\hat{\boldsymbol{n}}$. Its permittivity is denoted $\epsilon^{\prime}$, and its permeability $\mu^{\prime}$. It is embedded in a medium (e.g., vacuum) with permittivity $\epsilon$ and permeability $\mu$. When an incident electromagnetic field $\boldsymbol{e}^{\text {inc }}, \boldsymbol{h}^{\text {inc }}$ illuminates $\Omega$, the equivalent electric and magnetic current densities $\boldsymbol{j}, \boldsymbol{m}$ on $\Gamma$ satisfy the TD-PMCHWT equation:

$$
\left(\begin{array}{cc}
\frac{1}{\eta} \mathcal{T}+\frac{1}{\eta^{\prime}} \mathcal{T}^{\prime} & \mathcal{K}+\mathcal{K}^{\prime} \\
-\mathcal{K}-\mathcal{K}^{\prime} & \eta \mathcal{T}+\eta^{\prime} \mathcal{T}^{\prime}
\end{array}\right)\left(\begin{array}{c}
\boldsymbol{m} \\
\boldsymbol{j}
\end{array}\right)=-\left(\begin{array}{c}
\hat{\boldsymbol{n}} \times \boldsymbol{h}^{\mathrm{inc}} \\
\hat{\boldsymbol{n}} \times \boldsymbol{e}^{\mathrm{inc}}
\end{array}\right),
$$

where $\mathcal{T}$ and $\mathcal{K}$ are, respectively, the EFIE and the MFIE operators in $\mathbb{R}^{3} \backslash \Omega$, and $\mathcal{T}^{\prime}$ and $\mathcal{K}^{\prime}$ are the EFIE and the MFIE operators in $\Omega$. Their definitions are left out for brevity, but can be found in e.g. [3].

In order to discretize (1), define

$$
\left(\begin{array}{c}
\boldsymbol{j}^{\prime} \\
\boldsymbol{m}^{\prime}
\end{array}\right):=\left(\begin{array}{cc}
\frac{1}{\eta} \mathcal{T}+\frac{1}{\eta^{\prime}} \mathcal{T}^{\prime} & \mathcal{K}+\mathcal{K}^{\prime} \\
-\mathcal{K}-\mathcal{K}^{\prime} & \eta \mathcal{T}+\eta^{\prime} \mathcal{T}^{\prime}
\end{array}\right)\left(\begin{array}{c}
\boldsymbol{m} \\
\boldsymbol{j}
\end{array}\right) .
$$

The unknowns $\boldsymbol{j}$ and $\boldsymbol{m}$ are discretized in space using the Rao-Wilton-Glisson (RWG) functions $\boldsymbol{f}_{l}(\boldsymbol{r})$, while $\boldsymbol{j}^{\prime}$ and $\boldsymbol{m}^{\prime}$ are discretized using BuffaChristiansen (BC) functions $\boldsymbol{g}_{l}(\boldsymbol{r})$ :

$$
\begin{aligned}
\boldsymbol{m}(\boldsymbol{r}, t) & =\sum_{l=1}^{N_{S}} \mathrm{~m}_{l}(t) \boldsymbol{f}_{l}(\boldsymbol{r}) \\
\boldsymbol{j}(\boldsymbol{r}, t) & =\sum_{l=1}^{N_{S}} \mathrm{j}_{l}(t) \boldsymbol{f}_{l}(\boldsymbol{r}) \\
\boldsymbol{m}^{\prime}(\boldsymbol{r}, t) & =\sum_{l=1}^{N_{S}} \mathrm{~m}_{l}^{\prime}(t) \boldsymbol{g}_{l}(\boldsymbol{r}) \\
\boldsymbol{j}^{\prime}(\boldsymbol{r}, t) & =\sum_{l=1}^{N_{S}} \mathrm{j}_{l}^{\prime}(t) \boldsymbol{g}_{l}(\boldsymbol{r}) .
\end{aligned}
$$

In order to obtain a relation between $(\mathrm{m}(t), \mathrm{j}(t))$ and $\left(\mathrm{m}^{\prime}(t), \mathrm{j}^{\prime}(t)\right),(2)$ is spatially tested with the rotated RWG functions $\hat{\boldsymbol{n}} \times \boldsymbol{f}_{m}(\boldsymbol{r})$ :

$$
\left(\begin{array}{c}
\mathbf{j}^{\prime}(t) \\
\mathrm{m}^{\prime}(t)
\end{array}\right)=\left(\begin{array}{cc}
\mathbf{G}_{f g}^{-1} & \mathbf{0} \\
\mathbf{0} & \mathbf{G}_{f g}^{-1}
\end{array}\right)\left(\begin{array}{ll}
\mathcal{Q}_{11} & \mathcal{Q}_{12} \\
\mathcal{Q}_{21} & \mathcal{Q}_{22}
\end{array}\right)\left(\begin{array}{c}
\mathrm{m}(t) \\
\mathrm{j}(t)
\end{array}\right),
$$

with the Gram matrix

$$
\left[\mathbf{G}_{f g}\right]_{m n}=\int_{\Gamma}\left(\hat{\boldsymbol{n}} \times \boldsymbol{f}_{m}(\boldsymbol{r})\right) \cdot \boldsymbol{g}_{n}(\boldsymbol{r}) d s .
$$

The operators $\mathcal{Q}_{i j}$ map a time-dependent vector of RWG expansion coefficients into a time-dependent vector of RWG testing coefficients.
In terms of $\mathrm{m}^{\prime}(t)$ and $\mathrm{j}^{\prime}(t)$, the TD-PMCHWT equation (1) becomes

$$
\left(\begin{array}{c}
\mathbf{j}^{\prime}(t) \\
\mathbf{m}^{\prime}(t)
\end{array}\right)=-\left(\begin{array}{cc}
\mathbf{G}_{f g}^{-1} & \mathbf{0} \\
\mathbf{0} & \mathbf{G}_{f g}^{-1}
\end{array}\right)\left(\begin{array}{c}
\mathrm{h}(t) \\
\mathrm{e}(t)
\end{array}\right),
$$

with

$$
\begin{aligned}
{[\mathrm{h}(t)]_{m} } & =\int_{\Gamma} \boldsymbol{f}_{m}(\boldsymbol{r}) \cdot \boldsymbol{h}^{\mathrm{inc}}(\boldsymbol{r}, t) d s \\
{[\mathrm{e}(t)]_{m} } & =\int_{\Gamma} \boldsymbol{f}_{m}(\boldsymbol{r}) \cdot \boldsymbol{e}^{\mathrm{inc}}(\boldsymbol{r}, t) d s .
\end{aligned}
$$

\section{RESCALING}

Define the following auxiliary unknowns:

$$
\begin{aligned}
\mathrm{x}(t) & =\left(\mathbf{P}^{\Lambda H}+\frac{1}{T_{0}} \partial_{t}^{-1} \mathbf{P}^{\Sigma}\right) \mathrm{m}(t) \\
\mathrm{y}(t) & =\left(\mathbf{P}^{\Lambda H}+\frac{1}{T_{0}} \partial_{t}^{-1} \mathbf{P}^{\Sigma}\right) \mathrm{j}(t) \\
\mathrm{x}^{\prime}(t) & =\left(\mathbb{P}^{\Sigma H}+\frac{1}{T_{0}} \partial_{t}^{-1} \mathbb{P}^{\Lambda}\right) \mathrm{m}^{\prime}(t) \\
\mathrm{y}^{\prime}(t) & \left.=\left(\mathbb{P}^{\Lambda}+\frac{1}{T_{0}} \partial_{t}^{-1} \mathbb{P}^{\Lambda}\right) \mathbf{j}^{\prime} t\right) .
\end{aligned}
$$

The projectors $\mathbf{P}^{\Lambda H}$ and $\mathbf{P}^{\Sigma}$ are defined in [2], and project an arbitrary RWG coefficient vector onto the space of (local and global) RWG loops, and onto the space of RWG stars, respectively. Likewise, the projectors $\mathbb{P}^{\Sigma H}$ and $\mathbb{P}^{\Lambda}$ project an arbitrary $\mathrm{BC}$ coefficient vector onto the space of (local and global) $\mathrm{BC}$ loops, and onto the space of $\mathrm{BC}$ stars, respectively. When applied to multiply connected geometries, these projectors do not require the detection or construction of global loops.

The quantity $T_{0}$ is a scaling factor with the dimension of time. It is introduced to obtain a scale invariant and dimensionally consistent equation, and will be set to $T_{0}=D / c$, where $D$ is the diameter of the scatterer, and $c$ is the speed of light in the surrounding medium.

In terms of the auxiliary unknowns, (7) becomes

$$
\left(\begin{array}{l}
\mathrm{y}^{\prime}(t) \\
\mathrm{x}^{\prime}(t)
\end{array}\right)=\left(\begin{array}{ll}
\mathcal{Q}_{11}^{\prime} & \mathcal{Q}_{12}^{\prime} \\
\mathcal{Q}_{21}^{\prime} & \mathcal{Q}_{22}^{\prime}
\end{array}\right)\left(\begin{array}{l}
\mathrm{x}(t) \\
\mathrm{y}(t)
\end{array}\right)
$$

with

$$
\begin{aligned}
\mathcal{Q}_{i j}^{\prime}=\left(\mathbb{P}^{\Sigma H}+\frac{1}{T_{0}} \partial_{t}^{-1} \mathbb{P}^{\Lambda}\right) \cdot \mathbf{G}_{f g}^{-1} \\
\cdot \mathcal{Q}_{i j} \cdot\left(\mathbf{P}^{\Lambda H}+T_{0} \partial_{t} \mathbf{P}^{\Sigma}\right)
\end{aligned}
$$

With (9), the semi-discrete qHP-PMCHWT equation is obtained:

$$
\left(\begin{array}{ll}
\mathcal{Q}_{11}^{\prime} & \mathcal{Q}_{12}^{\prime} \\
\mathcal{Q}_{21}^{\prime} & \mathcal{Q}_{22}^{\prime}
\end{array}\right)\left(\begin{array}{l}
x(t) \\
\mathrm{y}(t)
\end{array}\right)=-\left(\begin{array}{l}
\mathrm{h}^{\prime}(t) \\
\mathrm{e}^{\prime}(t)
\end{array}\right)
$$


where

$$
\begin{aligned}
& \mathrm{h}^{\prime}(t)=\left(\mathbb{P}^{\Sigma H}+\frac{1}{T_{0}} \partial_{t}^{-1} \mathbb{P}^{\Lambda}\right) \mathbf{G}_{f g}^{-1} \mathrm{~h}(t) \\
& \mathrm{e}^{\prime}(t)=\left(\mathbb{P}^{\Sigma H}+\frac{1}{T_{0}} \partial_{t}^{-1} \mathbb{P}^{\Lambda}\right) \mathbf{G}_{f g}^{-1} \mathrm{e}(t) .
\end{aligned}
$$

The rescaling strategy leading to (18) is fundamentally different from the one applied to the TDEFIE in [1]. For the TD-EFIE, the basis and testing spaces are rescaled. For the TD-PMCHWT equation, the basis and range spaces are rescaled, leading to the presence of the inverse Gram matrix $\mathbf{G}_{f g}^{-1}$. This is essential in order to correctly handle global topological loops.

\section{TEMPORAL DISCRETIZATION}

In order to construct a numerical solution to the semi-discrete qHP-PMCHWT equation (18), it must be discretized in time. For this, a temporal Galerkin method is used. The correct choice of basis and testing functions is essential for the stability of the MOT scheme. Following [1], the loop and star parts of the auxiliary unknowns are expanded in different basis functions:

$$
\begin{aligned}
& \mathbf{x}(t)=\sum_{i=1}^{N_{T}}\left(p(t-i \Delta t) \mathbf{P}^{\Lambda H}+h(t-i \Delta t) \mathbf{P}^{\Sigma}\right) \mathbf{x}^{i} \\
& \mathbf{y}(t)=\sum_{i=1}^{N_{T}}\left(p(t-i \Delta t) \mathbf{P}^{\Lambda H}+h(t-i \Delta t) \mathbf{P}^{\Sigma}\right) \mathbf{y}^{i}
\end{aligned}
$$

where $p(t)$ are the pulse functions and $h(t)$ are the hat functions, as defined in [1].

Next, the following temporal Galerkin test procedure is applied to both lines of (18):

$$
\begin{gathered}
\int_{\mathbb{R}}\left(\frac{1}{\Delta t} p(t-j \Delta t) \mathbb{P}^{\Sigma H}+\delta(t-j \Delta t) \mathbb{P}^{\Lambda}\right) \\
\cdot(\text { Equation }(18)) d t,
\end{gathered}
$$

for $j=1,2, \ldots, N_{T}$, where $\delta(t)$ is the Dirac delta distribution. This yields the qHP-PMCHWT equation:

$$
\begin{aligned}
& -\left(\begin{array}{ll}
\mathbf{Q}_{11}^{\prime 0} & \mathbf{Q}_{12}^{\prime 0} \\
\mathbf{Q}_{21}^{\prime 0} & \mathbf{Q}_{22}^{\prime 0}
\end{array}\right)\left(\begin{array}{l}
\mathrm{x}^{j} \\
\mathbf{y}^{j}
\end{array}\right) \\
= & \sum_{i=1}^{j}\left(\begin{array}{ll}
\mathbf{Q}_{11}^{\prime i} & \mathbf{Q}_{12}^{\prime i} \\
\mathbf{Q}_{21}^{\prime i} & \mathbf{Q}_{22}^{\prime i}
\end{array}\right)\left(\begin{array}{l}
\mathrm{x}^{j-i} \\
\mathbf{y}^{j-i}
\end{array}\right)+\left(\begin{array}{c}
\mathbf{h}^{j} \\
\mathrm{e}^{j}
\end{array}\right) .
\end{aligned}
$$

Equation (24) can be solved successively for $j=$ $1,2,3$, etcetera. This is the marching-on-in-time algorithm.

In contrast to the standard TD-PMCHWT equation, the system (24) does not support static regime solutions that can become unstable due to numerical quadrature errors. Furthermore, the qHPPMCHWT equation is immune to low frequency breakdown: it remains well-conditioned for large time step sizes.

\section{NUMERICAL RESULTS}

In order to assess the perfomance of the qHPPMCHWT equation, consider the square torus depicted in Fig. 1. The medium inside the torus is characterized by $\epsilon^{\prime}=2 \epsilon_{0}$ and $\mu^{\prime}=\mu_{0}$. The surrounding medium has material parameters $\epsilon=\epsilon_{0}$ and $\mu=\mu_{0}$. This structure is illuminated by a Gaussian wave.

This scattering problem is solved numerically using the MOT algorithm applied to both the standard TD-PMCHWT equation and the qHPPMCHWT equation. The time step size is chosen as $c \Delta t=2 \mathrm{~m}$. The triangle mesh on which the spatial basis functions are defined contains 448 faces.

The current density in an arbitrary point is shown in Fig. 2. The two simulation techniques match for $c t<100 \mathrm{~m}$, but after that, the current obtained from the standard TD-PMCHWT equation grows exponentially: the simulation method is unstable. The qHP-PMCHWT equation, on the other hand, remains stable.

Next, a stability analysis is performed as in [4]: the stability of the MOT algorithm is defined by the eigenvalues of the so-called companion matrix. Eigenvalues located outside the unit circle indicate instability, whereas eigenvalues equal to one correspond to static regime solutions.

The eigenvalues of the companion matrix for the standard TD-PMCHWT equation are shown in Fig. 3, left. Part of the eigenvalues are scattered around 1 . When more accurate numerical quadrature rules are used, these eigenvalues contract towards 1 . However, in any realistic situation, there are still a number of eigenvalues located outside the unit circle. As a result, the simulation method is unstable. The eigenvalues for the qHP-PMCHWT equations are shown in Fig. 3, right. Here, all eigenvalues are located inside the unit circle. In particular, no cluster of eigenvalues is present around 1 .

Finally, the condition number of the MOT system matrix is computed for time steps ranging from $c \Delta t=1 \mathrm{~m}$ up to $c \Delta t=1024 \mathrm{~m}$ (Fig. 4). The condition number of the standard TD-PMCHWT equation grows proportionally to $\Delta t^{2}$. This is low frequency breakdown. The condition number of the qHP-PMCHWT equation, on the other hand, remains constant. This shows that the qHPPMCHWT equation does not suffer from low frequency breakdown. 


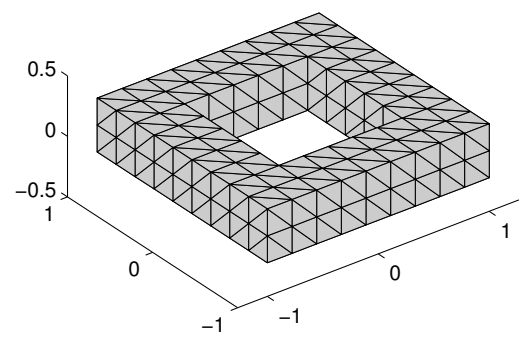

Figure 1: A square torus with $\epsilon^{\prime}=2 \epsilon_{0}, \mu^{\prime}=\mu_{0}$ is discretized using 448 triangles.

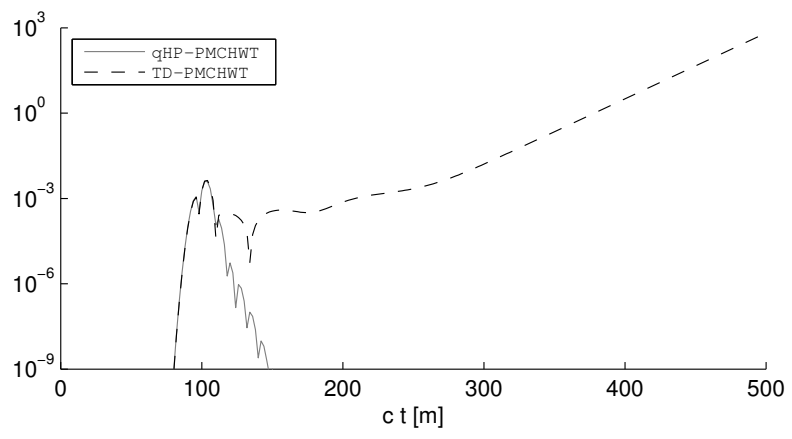

Figure 2: The current obtained from a standard TD-PMCHWT simulation (dashed line), and from a aHP-PMCHWT simulation (solid line).
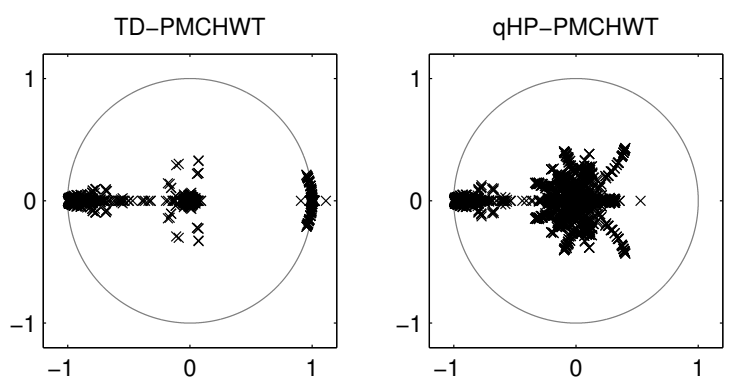

Figure 3: Stability analysis of the TD-PMCHWT equation (left) and the qHP-PMCHWT equation (right), as in [4].

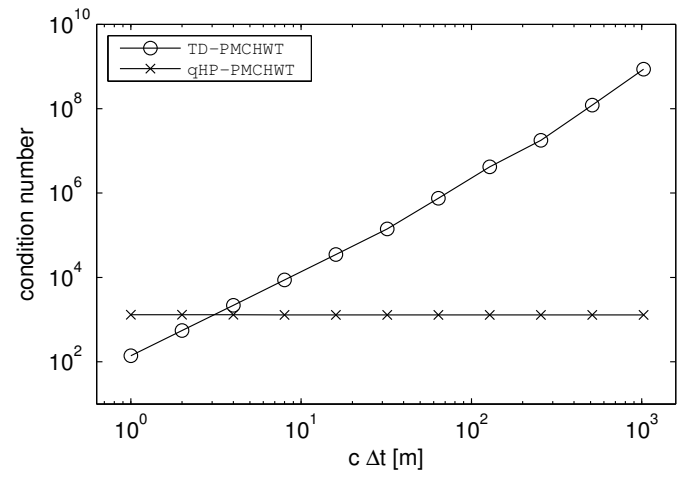

Figure 4: Condition number of the MOT system matrix as a function of the time step size $\Delta t$.

\section{CONCLUSIONS}

The time domain PMCHWT equation suffers from low frequency breakdown and DC instability. In this contribution, both issues are resolved by separating the equation's quasi-Helmholtz components using projector operators and integrating or differentiating them with respect to time. By applying a suitable temporal Galerkin method to each component, a system of discrete equations is obtained which can be solved using the marching-on-in-time method. This solution method is stable even in the presence of moderate quadrature errors, and remains well-conditioned even for very large time steps.

\section{Acknowledgments}

The work of Y. Beghein was supported by a doctoral grant from the Agency for Innovation by Science and Technology in Flanders (IWT).

\section{References}

[1] Y. Beghein, K. Cools, and F. Andriulli, "A DC stable and large time step well-balanced TDEFIE based on quasi-Helmholtz projectors," IEEE Transactions on Antennas and Propagation, vol. 63, no. 7, July 2015.

[2] F. Andriulli, K. Cools, I. Bogaert, and E. Michielssen, "On a well-conditioned electric field integral operator for multiply connected geometries," IEEE Transactions on Antennas and Propagation, vol. 61, no. 4, pp. 2077-2087, April 2013.

[3] Y. Beghein, K. Cools, H. Bagci, and D. De Zutter, "A space-time mixed galerkin marching-onin-time scheme for the time-domain combined field integral equation," IEEE Transactions on Antennas and Propagation, vol. 61, no. 3, pp. 1228-1238, March 2013.

[4] F. Andriulli, K. Cools, F. Olyslager, and E. Michielssen, "Time domain Calderón identities and their application to the integral equation analysis of scattering by PEC objects part II: Stability," IEEE Transactions on Antennas and Propagation, vol. 57, no. 8, pp. 2365-2375, Aug 2009. 